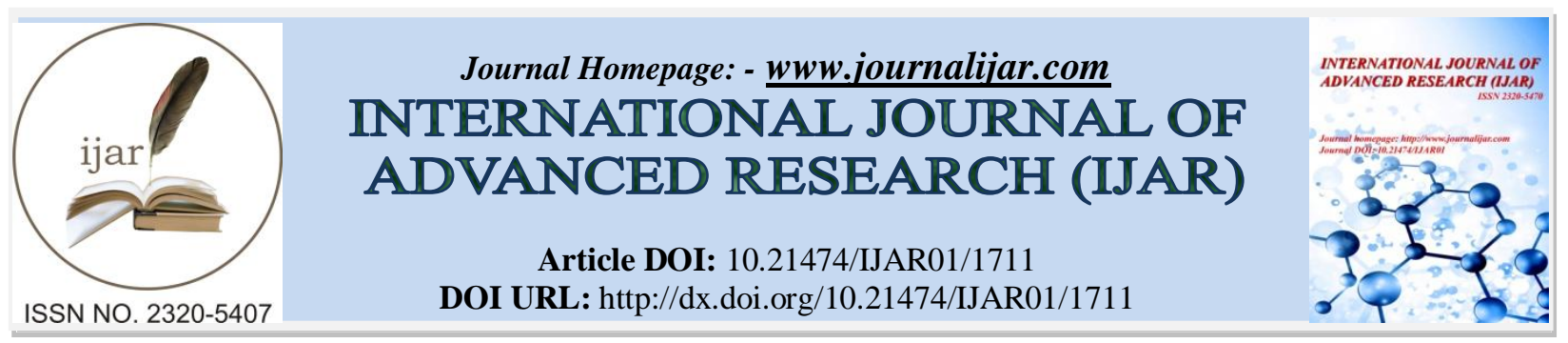

RESEARCH ARTICLE

\title{
Results on the Group Inverse for Block Matrices in Minkowski Space $\mathcal{M}$.
}

D. Krishnaswamy and Tasaduq Hussain Khan.

Department of Mathematics, Annamalai University, Annamalainagar - 608 002, India.

\section{Manuscript Info}

Manuscript History

Received: 12 July 2016

Final Accepted: 19 August 2016

Published: September 2016

\section{Abstract}

In this paper, existence and the representation of the group inverse for block matrix $M=\left(\begin{array}{ll}P^{\sim} & P^{\sim} \\ Q^{\sim} & 0\end{array}\right)\left(P, Q \in K^{n \times n},\left(P^{\sim}\right)^{2}=P^{\sim}\right)$ over skew fields in Minkowski space $\mathcal{M}$ is studied. The existence and the representation of the group inverse for other block matrices in Minkowski space $\mathcal{M}$ is also given.

Key words:-

Block matrix,

Group inverse,

Minkowski adjoint,

Minkowski Space.

Copy Right, IJAR, 2016,. All rights reserved.

\section{Introduction:-}

Suppose $K$ is a skew field. Let $K^{n \times n}$ denote the set of all matrices over $K$. For $A \in K^{n \times n}$, the matrix $X \in K^{n \times n}$ is said to be the group inverse of $A$, if it holds that

$$
A X A=A, \quad X A X=X, \quad A X=X A
$$

Denote by $X=A^{\#}$. By Zhuang (1987), if $X=A^{\#}$ exists and it is unique.

Kilicman et al., (2008) extend some results for the weighted Moore-Penrose inverse $A_{M, N}^{\dagger}$ in Hilbert Space to the weighted Minkowski inverse $A_{M, N}^{\oplus}$ of an arbitrary rectangle matrix $A \in M_{m, n}$ in Minkowski spaces $\mu$. Hanifa Zekraoui et al., (2013) introduce some new algebraic and topological properties of the Minkowski inverse $A^{\oplus}$ of an arbitrary matrix $A \in M_{m, n}$ in Minkowski space $\mu$. This paper also shows that Minkowski inverse $A^{\oplus}$ in a Minkowski space and Moore-Penrose inverse $A^{\dagger}$ in a Hilbert Space are different in many properties such as the existence, continuity, norm and SVD, and some new conditions like existence, continuity and reverse order law of the Minkowski inverse are also given. Meenakshi (2000) introduced the concept of range symmetric matrix and the existence of the Minkowski inverse of a range symmetric matrix in Minkowski space $\mathcal{M}$. Meenakshi et al., (2006) studied the necessary and sufficient conditions for the product of range symmetric matrices of rank $r$ to be range symmetric in Minkowski space $\mathcal{M}$.

In this paper the notations $A^{*}$, and $A^{\sim}$ stands for conjugate transpose and Minkowski adjoint of a matrix $A$ respectively, $I_{n}$ denote the identity matrix of order $n \times n$. Let $C^{n}$ be the space of complex $n$-tuples and we shall index them from 0 to $n-1$, that is $u=\left(u_{0}, u_{1}, u_{2}, \ldots, u_{n-1}\right)$. Let $G$ be the Minkowski metric tensor defined by

$G u=\left(u_{0},-u_{1},-u_{2}, \ldots,-u_{n-1}\right)$.

And the Minkowski metric matrix is defined by

$G=\left[\begin{array}{ll}1 & 0 \\ 0 & -I_{n-1}\end{array}\right] ; \quad G^{*}=G ; G^{2}=I_{n}$. 
In [15] the Minkowski inner product on $C^{n}$ is defined by $(u, v)=[u, G v]$, where [.,.] denotes the conventional Hilbert (unitary) space inner product. A space with Minkowski inner product is called a Minkowski space (Krishnaswamy et al., 2013). We establish the existence and the representation of the group inverse for block matrix $\left(\begin{array}{ll}P^{\sim} & P^{\sim} \\ Q^{\sim} & 0\end{array}\right)\left(P, Q \in K^{n \times n},\left(P^{\sim}\right)^{2}=P^{\sim}\right)$ in Minkowski space.

\section{Definition:-}

For any $P \in C^{n \times n}$, the Minkowski adjoint of $P$ denoted by $P^{\sim}$ is defined as $P=G P^{*} G$, where $P^{*}$ is the usual Hermitian adjoint and $G$ the Minkowski metric matrix of order $n$.

\section{Some Lemmas:-}

Lemma 2.1. Let $P \in K^{n \times n}$, if $\left(P^{\sim}\right)^{2}=P^{\sim}$, then there is a unitary matrix $A \in K^{n \times n}$ such that $P^{\sim}=\left(A^{*}\right)^{\sim}\left(\begin{array}{ll}I_{r} & 0 \\ 0 & 0\end{array}\right) A^{\sim}$, where $r=\operatorname{rank}\left(P^{\sim}\right), I_{r}$ is the $r \times r$ identity matrix, $I_{r} \in K^{n \times n}$.

Proof. Let $P \in K^{n \times n}$ then there are unitary matrices $A_{1}, A_{2}$ such that

$$
P=A_{1}\left(\begin{array}{cc}
I_{r} & 0 \\
0 & 0
\end{array}\right) A_{2} \Rightarrow P^{*}=A_{2}^{*}\left(\begin{array}{cc}
I_{r} & 0 \\
0 & 0
\end{array}\right) A_{1}^{*} \Rightarrow P^{\sim}=A_{2}^{\tilde{2}}\left(\begin{array}{cc}
I_{r} & 0 \\
0 & 0
\end{array}\right) A_{1}^{\tilde{1}}=A_{2}^{\tilde{2}}\left(\begin{array}{cc}
I_{r} & 0 \\
0 & 0
\end{array}\right) A_{1} A_{2}^{\tilde{2}}\left(A_{2}^{*}\right)^{\sim}
$$

Let $A_{1}^{\sim} A_{2}^{\sim}=\left(\begin{array}{ll}P_{1} & P_{2} \\ P_{3} & P_{4}\end{array}\right)$ then $P^{\sim}=A_{2}^{\sim}\left(\begin{array}{cc}P_{1} & P_{2} \\ 0 & 0\end{array}\right)\left(A_{2}^{*}\right)^{\sim}$

Since $\left(P^{\sim}\right)^{2}=P^{\sim}$, so we have $P_{1}\left(\begin{array}{ll}P_{1} & P_{2}\end{array}\right)=\left(\begin{array}{ll}P_{1} & P_{2}\end{array}\right)$ because $\left(\begin{array}{ll}P_{1} & P_{2}\end{array}\right)$ is full row rank, then $P_{1}=I_{r}$.

Let $A^{\sim}=A_{2}^{\sim}\left(\begin{array}{cc}I_{r} & -P_{2} \\ 0 & I_{n-r}\end{array}\right)$ then $P^{\sim}=A^{\sim}\left(\begin{array}{cc}I_{r} & 0 \\ 0 & 0\end{array}\right)\left(A^{*}\right)^{\sim}$

Lemma 2.2 Let $P, Q \in K^{n \times n}$, if $\left(P^{\sim}\right)^{2}=P^{\sim}, \operatorname{rank}\left(P^{\sim}\right)=r, \operatorname{rank}\left(Q^{\sim}\right)=\operatorname{rank}\left(Q^{\sim} P^{\sim} Q^{\sim}\right)$ then there is a unitary matrix $A \in K^{n \times n}$, such that $Q^{\sim}=A^{\sim}\left(\begin{array}{ll}Q_{1}^{*} & -Q_{1}^{*} X \\ -Y Q_{1}^{*} & Y Q_{1}^{*} X\end{array}\right)\left(A^{*}\right)^{\sim}$ and $\left(Q_{1}^{*}\right)^{\#}$ exists where $Q_{1}^{*} \in K^{n \times n}, X \in K^{r \times(n-r)}$, $Y \in K^{(n-r) \times r}$.

Proof. Since $\left(P^{\sim}\right)^{2}=P^{\sim}$, by Lemma 2.1 there is a unitary matrix $A \in K^{n \times n}$, such that

$$
P^{\sim}=A^{\sim}\left(\begin{array}{cc}
I_{r} & 0 \\
0 & 0
\end{array}\right)\left(A^{*}\right)^{\sim}, \quad Q^{\sim}=A^{\sim}\left(\begin{array}{ll}
Q_{1}^{*} & -Q_{3}^{*} \\
-Q_{2}^{*} & Q_{4}^{*}
\end{array}\right)\left(A^{*}\right)^{\sim}
$$

where $Q_{1}^{*} \in K^{r \times r}, Q_{2}^{*} \in K^{(n-r) \times r}, Q_{3}^{*} \in K^{r \times(n-r)}, Q_{4}^{*} \in K^{(n-r) \times(n-r)}$

Then

From

$$
P^{\sim} Q^{\sim}=A^{\sim}\left(\begin{array}{ll}
Q_{1}^{*} & -Q_{3}^{*} \\
0 & 0
\end{array}\right)\left(A^{*}\right)^{\sim}, \quad Q^{\sim} P^{\sim}=A^{\sim}\left(\begin{array}{ll}
Q_{1}^{*} & 0 \\
-Q_{2}^{*} & 0
\end{array}\right)\left(A^{*}\right)^{\sim}
$$

we have

$$
\operatorname{rank}\left(Q^{\sim}\right)=\operatorname{rank}\left(Q^{\sim} P^{\sim} Q^{\sim}\right) \leq \operatorname{rank}\left(Q^{\sim} P^{\sim}\right) \leq \operatorname{rank}\left(Q^{\sim}\right)
$$

From

$$
\operatorname{rank}\left(Q^{\sim}\right)=\operatorname{rank}\left(Q^{\sim} P^{\sim}\right)
$$

we get

$$
\operatorname{rank}\left(Q^{\sim}\right)=\operatorname{rank}\left(Q^{\sim} P^{\sim} Q^{\sim}\right) \leq \operatorname{rank}\left(P^{\sim} Q^{\sim}\right) \leq \operatorname{rank}\left(Q^{\sim}\right)
$$

Thus

$$
\operatorname{rank}\left(Q^{\sim}\right)=\operatorname{rank}\left(P^{\sim} Q^{\sim}\right)
$$

$$
\operatorname{rank}\left(P^{\sim}\right)=\operatorname{rank}\left(Q^{\sim} P^{\sim}\right)=\operatorname{rank}\left(Q^{\sim}\right)=\operatorname{rank}\left(P^{\sim} Q^{\sim}\right)
$$

Since from $\operatorname{rank}\left(Q^{\sim}\right)=\operatorname{rank}\left(P^{\sim} Q^{\sim}\right)$, we have

$$
Q_{2}^{*}=Y Q_{1}^{*}, \quad Q_{4}^{*}=Y Q_{3}^{*}, \quad Y \in K^{(n-r) \times r}
$$

and from $\operatorname{rank}\left(Q^{\sim}\right)=\operatorname{rank}\left(Q^{\sim} P^{\sim}\right)$, we get

So, we get

$$
Q_{3}^{*}=Q_{1}^{*} X, \quad Q_{4}^{*}=Q_{2}^{*} X=Y Q_{1}^{*} X, \quad X \in K^{r \times(n-r)}
$$

$$
Q^{\sim}=A^{\sim}\left(\begin{array}{ll}
Q_{1}^{*} & -Q_{1}^{*} X \\
-Y Q_{1}^{*} & Y Q_{1}^{*} X
\end{array}\right)\left(A^{*}\right)^{\sim}
$$

We have $\operatorname{rank}\left(Q_{1}^{*}\right)=\operatorname{rank}\left(Q_{1}^{*}\right)^{2}=\operatorname{rank}\left(Q^{\sim}\right)$, that is $\left(Q_{1}^{*}\right)^{\#}$ exists.

Lemma 2.3 Let $P \in K^{r \times r}, Q \in K^{(n-r) \times r}, M=\left(\begin{array}{ll}P^{\sim} & 0 \\ Q^{\sim} & 0\end{array}\right) \in K^{n \times n}$. Then the group inverse of $M$ exists in $\mathcal{M}$ if and only if the group inverse of $P^{\sim}$ exists in $\mathcal{M}$ and $\operatorname{rank}\left(P^{\sim}\right)=\operatorname{rank}\left(\begin{array}{l}P^{\sim} \\ Q^{\sim}\end{array}\right)$. If the group inverse of $M$ exists in $\mathcal{M}$, then 


$$
M^{\#}=\left(\begin{array}{ll}
\left(P^{\sim}\right)^{\#} & 0 \\
Q^{\sim}\left(\left(P^{\sim}\right)^{\#}\right)^{2} & 0
\end{array}\right)
$$

Proof. Since $M=\left(\begin{array}{ll}P^{\sim} & 0 \\ Q^{\sim} & 0\end{array}\right)$, suppose group inverse of $P^{\sim}$ exists in $\mathcal{M}$ and $\operatorname{rank}\left(P^{\sim}\right)=\operatorname{rank}\left(\begin{array}{l}P^{\sim} \\ Q^{\sim}\end{array}\right)$. Now $\operatorname{rank}(M)=\operatorname{rank}\left(\begin{array}{ll}P^{\sim} & 0 \\ Q^{\sim} & 0\end{array}\right)=\operatorname{rank}\left(\begin{array}{c}P^{\sim} \\ Q^{\sim}\end{array}\right)=\operatorname{rank}\left(P^{\sim}\right) . \operatorname{But} \operatorname{rank}\left(P^{\sim}\right)=\operatorname{rank}\left(P^{\sim}\right)^{2}$ as $\left(P^{\sim}\right)^{\#}$ exists $\Rightarrow \operatorname{rank}(M)=\operatorname{rank}\left(M^{2}\right)$. Therefore $M^{\#}$ exists in $\mathcal{M}$.

Conversely, suppose the group inverse of $M$ exists in $\mathcal{M}$, then it satisfies the following conditions, (i) $M M^{\#} M=M$, (ii) $M^{\#} M M^{\#}=M^{\#}$ and (iii) $M M^{\#}=M^{\#} M$. Also $\operatorname{rank}(M)=\operatorname{rank}\left(\begin{array}{ll}P^{\sim} & 0 \\ Q^{\sim} & 0\end{array}\right)=\operatorname{rank}\left(\begin{array}{l}P^{\sim} \\ Q^{\sim}\end{array}\right) \Rightarrow$ $\operatorname{rank}\left(P^{\sim}\right)=\operatorname{rank}\left(\begin{array}{l}P^{\sim} \\ Q^{\sim}\end{array}\right)$.

Let $M^{\#}=X=\left(\begin{array}{ll}\left(P^{\sim}\right)^{\#} & 0 \\ Q^{\sim}\left(\left(P^{\sim}\right)^{\#}\right)^{2} & 0\end{array}\right)$ then,

(i)

(ii)

$$
\begin{aligned}
M X M & =\left(\begin{array}{ll}
P^{\sim} & 0 \\
Q^{\sim} & 0
\end{array}\right)\left(\begin{array}{ll}
\left(P^{\sim}\right)^{\#} & 0 \\
Q^{\sim}\left(\left(P^{\sim}\right)^{\#}\right)^{2} & 0
\end{array}\right)\left(\begin{array}{ll}
P^{\sim} & 0 \\
Q^{\sim} & 0
\end{array}\right) \\
& =\left(\begin{array}{ll}
P^{\sim}\left(P^{\sim}\right)^{\#} P^{\sim} & 0 \\
Q^{\sim}\left(P^{\sim}\right)^{\#} P^{\sim} & 0
\end{array}\right) \\
& =\left(\begin{array}{ll}
P^{\sim} & 0 \\
Q^{\sim} & 0
\end{array}\right) \\
M X M & =M
\end{aligned}
$$

$$
\begin{aligned}
X M X & =\left(\begin{array}{ll}
\left(P^{\sim}\right)^{\#} & 0 \\
Q^{\sim}\left(\left(P^{\sim}\right)^{\#}\right)^{2} & 0
\end{array}\right)\left(\begin{array}{ll}
P^{\sim} & 0 \\
Q^{\sim} & 0
\end{array}\right)\left(\begin{array}{ll}
\left(P^{\sim}\right)^{\#} & 0 \\
Q^{\sim}\left(\left(P^{\sim}\right)^{\#}\right)^{2} & 0
\end{array}\right) \\
& =\left(\begin{array}{ll}
\left(P^{\sim}\right)^{\#} P^{\sim}\left(P^{\sim}\right)^{\#} & 0 \\
Q^{\sim}\left(\left(P^{\sim}\right)^{\#}\right)^{2} P^{\sim}\left(P^{\sim}\right)^{\#} & 0
\end{array}\right) \\
& =\left(\begin{array}{ll}
\left(P^{\sim}\right)^{\#} & 0 \\
Q^{\sim}\left(\left(P^{\sim}\right)^{\#}\right)^{2} & 0
\end{array}\right) \\
X M X & =X
\end{aligned}
$$

$$
\begin{aligned}
M X & =\left(\begin{array}{ll}
P^{\sim} & 0 \\
Q^{\sim} & 0
\end{array}\right)\left(\begin{array}{ll}
\left(P^{\sim}\right)^{\#} & 0 \\
Q^{\sim}\left(\left(P^{\sim}\right)^{\#}\right)^{2} & 0
\end{array}\right) \\
& =\left(\begin{array}{ll}
P^{\sim}\left(P^{\sim}\right)^{\#} & 0 \\
Q^{\sim}\left(P^{\sim}\right)^{\#} & 0
\end{array}\right) \\
X M & =\left(\begin{array}{ll}
\left(P^{\sim}\right)^{\#} & 0 \\
Q^{\sim}\left(\left(P^{\sim}\right)^{\#}\right)^{2} & 0
\end{array}\right)\left(\begin{array}{ll}
P^{\sim} & 0 \\
Q^{\sim} & 0
\end{array}\right) \\
& =\left(\begin{array}{ll}
P^{\sim}\left(P^{\sim}\right)^{\#} & 0 \\
Q^{\sim}\left(P^{\sim}\right)^{\#} & 0
\end{array}\right) \\
X M & =M X .
\end{aligned}
$$

Lemma 2.4 Let $P \in K^{r \times r}, Q \in K^{r \times(n-r)}$, and $M=\left(\begin{array}{cc}P^{\sim} & Q^{\sim} \\ 0 & 0\end{array}\right) \in K^{n \times n}$. Then the group inverse of $M$ exists in $\mathcal{M}$ if and only if the group inverse of $P^{\sim}$ exists in $\mathcal{M}$ and $\operatorname{rank}\left(P^{\sim}\right)=\operatorname{rank}\left(P^{\sim} \quad Q^{\sim}\right)$. If the group inverse of $M$ exists in $\mathcal{M}$, then,

Proof. The proof is same as Lemma 2.3

$$
M^{\#}=\left(\begin{array}{ll}
\left(P^{\sim}\right)^{\#} & \left(\left(P^{\sim}\right)^{\#}\right)^{2} Q^{\sim} \\
0 & 0
\end{array}\right)
$$

Lemma 2.5 Let $P, Q \in K^{n \times n}$, if $\left(P^{\sim}\right)^{2}=P^{\sim}, \operatorname{rank}\left(P^{\sim}\right)=r, \operatorname{rank}\left(Q^{\sim}\right)=\operatorname{rank}\left(Q^{\sim} P^{\sim} Q^{\sim}\right)$ then the following conclusions hold:

(i) $\left(Q^{\sim} P^{\sim}\right)^{\#} Q^{\sim} P^{\sim} Q^{\sim}=Q^{\sim}$; 
(ii) $P^{\sim}\left(P^{\sim} Q^{\sim}\right)^{\#}=\left(P^{\sim} Q^{\sim}\right)^{\#},\left(Q^{\sim} P^{\sim}\right)^{\#} P^{\sim}=\left(Q^{\sim} P^{\sim}\right)^{\#},\left(P^{\sim} Q^{\sim}\right)^{\#} P^{\sim}=P^{\sim}\left(Q^{\sim} P^{\sim}\right)^{\#},\left(Q^{\sim} P^{\sim}\right)^{\#} Q^{\sim}=Q^{\sim}\left(P^{\sim} Q^{\sim}\right)^{\#}$;

(iii) $\left(P^{\sim} Q^{\sim}\right)^{\#} P^{\sim} Q^{\sim} P^{\sim}\left(P^{\sim} Q^{\sim}\right)^{\#}=\left(P^{\sim} Q^{\sim}\right)^{\#}, P^{\sim}\left(Q^{\sim} P^{\sim}\right)^{\#}\left(P^{\sim} Q^{\sim}\right)^{\#} P^{\sim} Q^{\sim}=\left(P^{\sim} Q^{\sim}\right)^{\#}$;

(iv) $\left(Q^{\sim} P^{\sim}\right)^{\#} Q^{\sim} P^{\sim}\left(P^{\sim} Q^{\sim}\right)^{\#} P^{\sim}=\left(Q^{\sim} P^{\sim}\right)^{\#}, Q^{\sim}\left(P^{\sim} Q^{\sim}\right)^{\#} P^{\sim} Q^{\sim} P^{\sim}=Q^{\sim} P^{\sim}$.

Proof. Using Lemma 2.2, 2.3 and 2.4 we have

then

$$
P^{\sim} Q^{\sim}=\left(A^{*}\right)^{\sim}\left(\begin{array}{ll}
Q_{1}^{*} & -Q_{1}^{*} X \\
0 & 0
\end{array}\right) A^{\sim}, \quad Q^{\sim} P^{\sim}=\left(A^{*}\right)^{\sim}\left(\begin{array}{ll}
Q_{1}^{*} & 0 \\
-Y Q_{1}^{*} & 0
\end{array}\right) A^{\sim}
$$

$$
\left(P^{\sim} Q^{\sim}\right)^{\#}=\left(A^{*}\right)^{\sim}\left(\begin{array}{ll}
\left(Q_{1}^{*}\right)^{\#} & -\left(Q_{1}^{*}\right)^{\#} X \\
0 & 0
\end{array}\right) A^{\sim}, \quad\left(Q^{\sim} P^{\sim}\right)^{\#}=\left(A^{*}\right)^{\sim}\left(\begin{array}{ll}
\left(Q_{1}^{*}\right)^{\#} & 0 \\
-Y\left(Q_{1}^{*}\right)^{\#} & 0
\end{array}\right) A^{\sim}
$$

$$
\begin{aligned}
\left(Q^{\sim} P^{\sim}\right)^{\#} Q^{\sim} P^{\sim} Q^{\sim} & =\left(A^{*}\right)^{\sim}\left(\begin{array}{ll}
\left(Q_{1}^{*}\right)^{\#} & 0 \\
-Y\left(Q_{1}^{*}\right)^{\#} & 0
\end{array}\right) A^{\sim}\left(A^{*}\right)^{\sim}\left(\begin{array}{ll}
Q_{1}^{*} & 0 \\
-Y Q_{1}^{*} & 0
\end{array}\right) A^{\sim}\left(A^{*}\right)^{\sim}\left(\begin{array}{ll}
Q_{1}^{*} & -Q_{1}^{*} X \\
-Y Q_{1}^{*} & Y Q_{1}^{*} X
\end{array}\right) A^{\sim} \\
& =\left(A^{*}\right)^{\sim}\left(\begin{array}{ll}
Q_{1}^{*} & -Q_{1}^{*} X \\
-Y Q_{1}^{*} & Y Q_{1}^{*} X
\end{array}\right) A^{\sim} \\
& =Q^{\sim}
\end{aligned}
$$

Similarly (ii) - (iv) can be proved.

\section{Conclusion:-}

Theorem 3.1 Suppose $M=\left(\begin{array}{ll}P^{\sim} & P^{\sim} \\ Q^{\sim} & 0\end{array}\right)$ where $P, Q \in K^{n \times n},\left(P^{\sim}\right)^{2}=P^{\sim}, \operatorname{rank}\left(P^{\sim}\right)=r$, then

(i) $M^{\#}$ exists if and only if $\operatorname{rank}\left(Q^{\sim}\right)=\operatorname{rank}\left(Q^{\sim} P^{\sim} Q^{\sim}\right)$;

(ii) If $M^{\#}$ exists, then

$$
M^{\#}=\left(\begin{array}{ll}
P^{\sim}-\left(P^{\sim} Q^{\sim}\right)^{\#}+\left(P^{\sim} Q^{\sim}\right)^{\#} P^{\sim}-\left(P^{\sim} Q^{\sim}\right)^{\#} P^{\sim} Q^{\sim} P^{\sim} & P^{\sim}+\left(P^{\sim} Q^{\sim}\right)^{\#} P^{\sim}-\left(P^{\sim} Q^{\sim}\right)^{\#} P^{\sim} Q^{\sim} P^{\sim} \\
\left(Q^{\sim} P^{\sim}\right)^{\#} Q^{\sim}+\left(Q^{\sim} P^{\sim}\right)^{\#}\left(P^{\sim} Q^{\sim}\right)^{\#} P^{\sim} Q^{\sim}-\left(Q^{\sim} P^{\sim}\right)^{\#} & -\left(Q^{\sim} P^{\sim}\right)^{\#}
\end{array}\right)
$$

Proof. $(i) \operatorname{rank}(M)=\operatorname{rank}\left(\begin{array}{ll}P^{\sim} & P^{\sim} \\ Q^{\sim} & 0\end{array}\right)=\operatorname{rank}\left(\begin{array}{ll}0 & P^{\sim} \\ Q^{\sim} & 0\end{array}\right)=\operatorname{rank}\left(P^{\sim}\right)+\operatorname{rank}\left(Q^{\sim}\right)$.

$$
\begin{gathered}
\operatorname{rank}\left(M^{2}\right)=\operatorname{rank}\left(\begin{array}{ll}
P^{\sim}+P^{\sim} Q^{\sim} & P^{\sim} \\
Q^{\sim} P^{\sim} & Q^{\sim} P^{\sim}
\end{array}\right)=\operatorname{rank}\left(\begin{array}{ll}
P^{\sim} Q^{\sim} & P^{\sim} \\
0 & Q^{\sim} P^{\sim}
\end{array}\right)=\operatorname{rank}\left(\begin{array}{ll}
0 & P^{\sim} \\
Q^{\sim} P^{\sim} Q^{\sim} & Q^{\sim} P^{\sim}
\end{array}\right) \\
=\operatorname{rank}\left(\begin{array}{ll}
0 & P^{\sim} \\
Q^{\sim} P^{\sim} Q^{\sim} & 0
\end{array}\right)=\operatorname{rank}\left(P^{\sim}\right)+\operatorname{rank}\left(Q^{\sim} P^{\sim} Q^{\sim}\right) .
\end{gathered}
$$

$M^{\#}$ exists if and only if $\operatorname{rank}(M)=\operatorname{rank}\left(M^{2}\right)$, that is $\operatorname{rank}\left(Q^{\sim}\right)=\operatorname{rank}\left(Q^{\sim} P^{\sim} Q^{\sim}\right)$.

$$
\begin{aligned}
& \text { (ii) Let } X=\left(\begin{array}{ll}
Z_{1} & Z_{2} \\
Z_{3} & Z_{4}
\end{array}\right) \text { where, } \\
& Z_{1}=P^{\sim}-\left(P^{\sim} Q^{\sim}\right)^{\#}+\left(P^{\sim} Q^{\sim}\right)^{\#} P^{\sim}-\left(P^{\sim} Q^{\sim}\right)^{\#}-\left(P^{\sim} Q^{\sim}\right)^{\#} P^{\sim} Q^{\sim} P^{\sim} \text {; } \\
& Z_{2}=P^{\sim}+\left(P^{\sim} Q^{\sim}\right)^{\#} P^{\sim}-\left(P^{\sim} Q^{\sim}\right)^{\#} P^{\sim} Q^{\sim} P^{\sim} \text {; } \\
& Z_{3}=\left(Q^{\sim} P^{\sim}\right)^{\#} Q^{\sim}+\left(Q^{\sim} P^{\sim}\right)^{\#}\left(P^{\sim} Q^{\sim}\right)^{\#} P^{\sim} Q^{\sim}-\left(Q^{\sim} P^{\sim}\right)^{\#} \text {; } \\
& Z_{4}=-\left(Q^{\sim} P^{\sim}\right)^{\#}
\end{aligned}
$$

Then we will prove that the above matrix satisfies the three conditions of group inverse.

$$
\begin{aligned}
M X M & =\left(\begin{array}{ll}
P^{\sim} & P^{\sim} \\
Q^{\sim} & 0
\end{array}\right)\left(\begin{array}{ll}
Z_{1} & Z_{2} \\
Z_{3} & Z_{4}
\end{array}\right)\left(\begin{array}{ll}
P^{\sim} & P^{\sim} \\
Q^{\sim} & 0
\end{array}\right) \\
& =\left(\begin{array}{ll}
P^{\sim}\left(Z_{1}+Z_{2}\right) P^{\sim}+P^{\sim}\left(Z_{2}+Z_{4}\right) Q^{\sim} & P^{\sim}\left(Z_{1}+Z_{3}\right) P^{\sim} \\
Q^{\sim} Z_{1} P^{\sim}+Q^{\sim} Z_{2} Q^{\sim} & Q^{\sim} Z_{1} P^{\sim}
\end{array}\right)
\end{aligned}
$$

Using Lemma 2.5 we get from $(2,1)$ block of $M X M$

$$
\begin{aligned}
Q^{\sim} Z_{1} P^{\sim}+Q^{\sim} Z_{2} Q^{\sim}= & Q^{\sim}\left(P^{\sim}-\left(P^{\sim} Q^{\sim}\right)^{\#}+\left(P^{\sim} Q^{\sim}\right)^{\#} P^{\sim}-\left(P^{\sim} Q^{\sim}\right)^{\#} P^{\sim} Q^{\sim} P^{\sim}\right) P^{\sim}+Q^{\sim}\left(P^{\sim}+\left(P^{\sim} Q^{\sim}\right)^{\#} P^{\sim}\right. \\
& \left.-\left(P^{\sim} Q^{\sim}\right)^{\#} P^{\sim} Q^{\sim} P^{\sim}\right) Q^{\sim} \\
= & Q^{\sim} P^{\sim}-Q^{\sim}\left(P^{\sim} Q^{\sim}\right)^{\#} P^{\sim} Q^{\sim} P^{\sim}+Q^{\sim} P^{\sim} Q^{\sim}+Q^{\sim}\left(P^{\sim} Q^{\sim}\right)^{\#} P^{\sim} Q^{\sim} P^{\sim} Q^{\sim} \\
= & \left(Q^{\sim} P^{\sim}\right)^{\#} Q^{\sim} P^{\sim} Q^{\sim} \\
= & Q^{\sim}
\end{aligned}
$$

Similarly we can get

$$
\begin{aligned}
& P^{\sim}\left(Z_{1}+Z_{3}\right) P^{\sim}+P^{\sim}\left(Z_{2}+Z_{4}\right) Q^{\sim}=P^{\sim} ; \\
& P^{\sim}\left(Z_{1}+Z_{3}\right) P^{\sim}=P^{\sim} ; \\
& Q^{\sim} Z_{1} P^{\sim}=0
\end{aligned}
$$

Now 
Using Lemma 2.6 we get

$$
\begin{aligned}
X M X & =\left(\begin{array}{ll}
Z_{1} & Z_{2} \\
Z_{3} & Z_{4}
\end{array}\right)\left(\begin{array}{cc}
P^{\sim} & P^{\sim} \\
Q^{\sim} & 0
\end{array}\right)\left(\begin{array}{ll}
Z_{1} & Z_{2} \\
Z_{3} & Z_{4}
\end{array}\right) \\
& =\left(\begin{array}{ll}
Z_{1} P^{\sim} Z_{1}+Z_{2} Q^{\sim} Z_{1}+Z_{1} P^{\sim} Z_{3} & Z_{1} P^{\sim} Z_{2}+Z_{2} Q^{\sim} Z_{2}+Z_{1} P^{\sim} Z_{4} \\
Z_{3} P^{\sim} Z_{1}+Z_{4} Q^{\sim} Z_{1}+Z_{3} P^{\sim} Z_{3} & Z_{3} P^{\sim} Z_{3}+Z_{4} Q^{\sim} Z_{2}+Z_{3} P^{\sim} Z_{4}
\end{array}\right)
\end{aligned}
$$

$$
\begin{aligned}
Z_{1} P^{\sim} Z_{1}+Z_{2} Q^{\sim} Z_{1}+Z_{1} P^{\sim} Z_{3}= & Z_{1}+P^{\sim}\left(Q^{\sim} P^{\sim}\right)^{\#} Q^{\sim}+P^{\sim}\left(Q^{\sim} P^{\sim}\right)^{\#}\left(P^{\sim} Q^{\sim}\right)^{\#} P^{\sim} Q^{\sim}-P^{\sim}\left(Q^{\sim} P^{\sim}\right)^{\#} \\
& +\left(P^{\sim} Q^{\sim}\right)^{\#} P^{\sim} Q^{\sim} P^{\sim}\left(P^{\sim} Q^{\sim}\right)^{\#}-\left(P^{\sim} Q^{\sim}\right)^{\#} P^{\sim} Q^{\sim} P^{\sim}\left(P^{\sim} Q^{\sim}\right)^{\#} P^{\sim} \\
& +\left(P^{\sim} Q^{\sim}\right)^{\#} P^{\sim} Q^{\sim} P^{\sim}\left(P^{\sim} Q^{\sim}\right)^{\#} P^{\sim} Q^{\sim} P^{\sim}-\left(P^{\sim} Q^{\sim}\right)^{\#} P^{\sim} Q^{\sim} P^{\sim}\left(Q^{\sim} P^{\sim}\right)^{\#} Q^{\sim} \\
& -\left(P^{\sim} Q^{\sim}\right)^{\#} P^{\sim} Q^{\sim} P^{\sim}\left(Q^{\sim} P^{\sim}\right)^{\#}\left(P^{\sim} Q^{\sim}\right)^{\#} P^{\sim} Q^{\sim}+\left(P^{\sim} Q^{\sim}\right)^{\#} P^{\sim} Q^{\sim} P^{\sim}\left(Q^{\sim} P^{\sim}\right)^{\#} \\
& -\left(P^{\sim} Q^{\sim}\right)^{\#}+\left(P^{\sim} Q^{\sim}\right)^{\#} P^{\sim}-\left(P^{\sim} Q^{\sim}\right)^{\#} P^{\sim} Q^{\sim} P^{\sim} \\
= & Z_{1}+\left(P^{\sim} Q^{\sim}\right)^{\#} P^{\sim} Q^{\sim}+\left(P^{\sim} Q^{\sim}\right)^{\#}-\left(P^{\sim} Q^{\sim}\right)^{\#} P^{\sim}+\left(P^{\sim} Q^{\sim}\right)^{\#}-\left(P^{\sim} Q^{\sim}\right)^{\#} P^{\sim} \\
& +\left(P^{\sim} Q^{\sim}\right)^{\#} P^{\sim} Q^{\sim} P^{\sim}-\left(P^{\sim} Q^{\sim}\right)^{\#} P^{\sim} Q^{\sim}-\left(P^{\sim} Q^{\sim}\right)^{\#}+\left(P^{\sim} Q^{\sim}\right)^{\#} P^{\sim}-\left(P^{\sim} Q^{\sim}\right)^{\#} \\
& +\left(P^{\sim} Q^{\sim}\right)^{\#} P^{\sim}-\left(P^{\sim} Q^{\sim}\right)^{\#} P^{\sim} Q^{\sim} P^{\sim} \\
= & Z_{1}
\end{aligned}
$$

We can easily get$$
\begin{aligned}
& Z_{1} P^{\sim} Z_{2}+Z_{2} Q^{\sim} Z_{2}+Z_{1} P^{\sim} Z_{4}=Z_{2} \\
& Z_{3} P^{\sim} Z_{1}+Z_{4} Q^{\sim} Z_{1}+Z_{3} P^{\sim} Z_{3}=Z_{3} \\
& Z_{3} P^{\sim} Z_{3}+Z_{4} Q^{\sim} Z_{2}+Z_{3} Q^{\sim} Z_{4}=Z_{4}
\end{aligned}
$$

$Z_{3} P^{\sim} Z_{3}+Z_{4} Q^{\sim} Z_{2}+Z_{3} Q^{\sim} Z_{4}=Z_{4}$

Finally $M X=X M=\left(\begin{array}{ll}P^{\sim}-\left(P^{\sim} Q^{\sim}\right)^{\#} P^{\sim} Q^{\sim} P^{\sim}+\left(P^{\sim} Q^{\sim}\right)^{\#} P^{\sim} Q^{\sim} & P^{\sim}-\left(P^{\sim} Q^{\sim}\right)^{\#} P^{\sim} Q^{\sim} P^{\sim} \\ \left(Q^{\sim} P^{\sim}\right)^{\#} Q^{\sim} P^{\sim}-\left(Q^{\sim} P^{\sim}\right)^{\#} Q^{\sim} & \left(Q^{\sim} P^{\sim}\right)^{\#} Q^{\sim} P^{\sim}\end{array}\right)$

So we have $X=M^{\#}$.

Theorem 3.2 Suppose $M=\left(\begin{array}{ll}P^{\sim} & Q^{\sim} \\ P^{\sim} & 0\end{array}\right)$ where $P, Q \in K^{n \times n},\left(P^{\sim}\right)^{2}=P^{\sim}, \operatorname{rank}\left(P^{\sim}\right)=r$, then

(i) $M^{\#}$ exists if and only if $\operatorname{rank}\left(Q^{\sim}\right)=\operatorname{rank}\left(Q^{\sim} P^{\sim} Q^{\sim}\right)$;

(ii) If $M^{\#}$ exists, then

$M^{\#}=\left(\begin{array}{ll}P^{\sim}-\left(Q^{\sim} P^{\sim}\right)^{\#}+\left(P^{\sim} Q^{\sim}\right)^{\#} P^{\sim}-\left(P^{\sim} Q^{\sim}\right)^{\#} P^{\sim} Q^{\sim} P^{\sim} & \left(Q^{\sim} P^{\sim}\right)^{\#} Q^{\sim}+\left(Q^{\sim} P^{\sim}\right)^{\#}\left(P^{\sim} Q^{\sim}\right) P^{\sim} Q^{\sim}-\left(P^{\sim} Q^{\sim}\right)^{\#} \\ P^{\sim}+\left(P^{\sim} Q^{\sim}\right)^{\#} P^{\sim}-\left(P^{\sim} Q^{\sim}\right)^{\#} P^{\sim} Q^{\sim} P^{\sim} & -\left(P^{\sim} Q^{\sim}\right)^{\#}\end{array}\right)$

Proof. Proof is same as Theorem 3.1.

Theorem 3.3 If $\left(\begin{array}{cc}P^{\sim} & P^{\sim} \\ Q^{\sim} & 0\end{array}\right)^{\#}$ exists, where $P, Q \in K^{n \times n},\left(P^{\sim}\right)^{2}=P^{\sim}$, rank $\left(P^{\sim}\right)=r$, then $P^{\sim} Q^{\sim}$ and $Q^{\sim} P^{\sim}$ are similar.

Proof. Since $\left(\begin{array}{ll}P^{\sim} & P^{\sim} \\ Q^{\sim} & 0\end{array}\right)^{\#}$ exists, using Lemma 2.1 and Lemma 2.2 there is a unitary matrix $A \in K^{n \times n}$, such that

Hence

$$
\begin{aligned}
P^{\sim}=\left(A^{*}\right)^{\sim} & \left(\begin{array}{ll}
I_{r} & 0 \\
0 & 0
\end{array}\right) A^{\sim}, \quad Q^{\sim}=\left(A^{*}\right)^{\sim}\left(\begin{array}{ll}
Q_{1}^{*} & -Q_{1}^{*} X \\
-Y Q_{1}^{*} & Y Q_{1}^{*} X
\end{array}\right) A^{\sim} \\
P^{\sim} Q^{\sim} & =\left(A^{*}\right)^{\sim}\left(\begin{array}{ll}
Q_{1}^{*} & -Q_{1}^{*} X \\
0 & 0
\end{array}\right) A^{\sim} \\
& =\left(A^{*}\right)^{\sim}\left(\begin{array}{ll}
I_{r} & X \\
0 & I_{n-r}
\end{array}\right)\left(\begin{array}{ll}
Q_{1}^{*} & 0 \\
0 & 0
\end{array}\right)\left(\begin{array}{ll}
I_{r} & -X \\
0 & I_{n-r}
\end{array}\right) A^{\sim}
\end{aligned}
$$

$$
\begin{aligned}
Q^{\sim} P^{\sim} & =\left(A^{*}\right)^{\sim}\left(\begin{array}{lll}
Q_{1}^{*} & & 0 \\
-Y & Q_{1}^{*} & 0
\end{array}\right) A^{\sim} \\
& =\left(A^{*}\right)^{\sim}\left(\begin{array}{ll}
I_{r} & 0 \\
-Y & I_{n-r}
\end{array}\right)\left(\begin{array}{ll}
Q_{1}^{*} & 0 \\
0 & 0
\end{array}\right)\left(\begin{array}{ll}
I_{r} & 0 \\
Y^{\prime} & I_{n-r}
\end{array}\right) A^{\sim}
\end{aligned}
$$

So $P^{\sim} Q^{\sim}$ and $Q^{\sim} P^{\sim}$ are similar. 


\section{Refrences:-}

1. Kilicman, A., and Al-Zhour, Z., (2008), The representation and approximation for the weighted Minkowski inverse in Minkowski space, Mathematical and computer modelling 47:363-371.

2. Krishnaswamy, D., Punithavalli, G., (2013), The re-nnd definite solutions of the matrix equation $A X B=C$ in Minkowski space $\mathcal{M}$, Int. J. Fuzzy Math. Arch. 2:70-77.

3. Meenakshi, A.R., (2000), Range Symmetric Matrices in Minkowski Space, Bulletin of the Malaysian Mathematical Sciences Society, 23: 45-52.

4. Meenakshi, A.R. and Krishnaswamy, D., (2006), Product of Range Symmetric Block Matrices in Minkowski Space, Bulletin of the Malaysian Mathematical Sciences Society, 29(1): 59-68.

5. Zekraoui, H., Al-Zhour, Z., and Ozel, C., (2013), Some New Algebraic and Topological Properties of the Minkowski Inverse in the Minkowski Space, Hindawi Publishing Corporation, Article ID 765732, 6 pages.

6. Zhuang, W.J., (1987) Involuntary function and generalized inverses of matrices over skew fields, Northeast Math. 1:57-65. 